\title{
Considering the Use of the Term 'Rehab Potential' within in-patient Mental Health Services: Its Usefulness and the Risks Associated with Getting it Wrong-A Theoretical Perspective
}

\author{
Christopher Neil Edge $(\mathbb{D} \cdot$ Neil Tilston-Roberts
}

Received: 17 April 2020/ Accepted: 9 July 2020/Published online: 16 July 2020

(C) The Author(s) 2020

\begin{abstract}
Rehab potential' is a term that is frequently used within in-patient mental health services as means of predicting one's potential response to rehabilitation-focused interventions. However, there is no explicit and common understanding of the factors that contribute to concept of rehabilitation potential within the context of mental health rehabilitation, despite this being such a commonly used phrase. When accurate predictions are made about a person's perceived rehabilitation potential, it has the power to enhance a person's rehabilitation process. If these predictions are inaccurate, they can have negative consequences for the individuals involved. Consequences of inaccurately predicting an individual's rehabilitation potential can include people being denied access to rehabilitation services or being confined to years of care and/or more restrictive services that may not promote independence or recovery from mental illness as effectively as rehabilitation-focused services. This can have significant implications for these individuals such as reduced feelings of well-being and quality of life. In other medical fields there is evidence that judgments of
\end{abstract}

C. N. Edge ( $\square)$

School of Health Sciences, University of Liverpool, Liverpool, UK

e-mail: c.edge@liverpool.ac.uk

N. Tilston-Roberts

School of Social and Life Sciences, Wrexham Glyndwr

University, Wrexham, UK rehabilitation potential can also have negative implications for the members of staff expected to make these decisions on behalf of service users.

Keywords Mental health · Rehabilitation potential . Rehabilitation · Treatment planning · Treatment trajectory

\section{Introduction}

Within in-patient mental health services, it is not uncommon to hear phrases such as "this service user lacks rehab potential" when clinicians are discussing service users in the context of particular situations. These situations can include for example, during the pre-admission process, where potential candidates for admission to services are evaluated or when attempting to determine the appropriate treatment focus for an individual.

It is common practice for rehabilitation-focused, inpatient mental health services to employ a method of screening potential service users in order to determine those candidates most suitable for admission to these services, based upon the potential benefit to the service user [10]. In a UK-wide survey, Killaspy et al. [6] outlined that a variety of processes were employed by services when determining suitable individuals for admission, with no standardized method existing 
across the sector. Services are free to carry out screening in a manner that they deem suitable and subsequently, make decisions regarding the suitability of potential service users for admission to their particular service. Following these assessments, the aforementioned phrase, "this service user lacks rehab potential" will often rear its head when clinicians discuss and justify reasons not to admit a service user to a particular service.

Sinclair and Dickinson [12] broadly defined rehabilitation as a process aiming to restore personal autonomy within aspects of one's daily living. Wade [14] has since extended this as "a problem-solving process, framed in the context of the holistic biopsychosocial model of illness, delivered in a personcentred way." Understanding the need for a more universally accepted definition of rehabilitation in the field of mental health rehabilitation, Killaspy et al. [6] conducted a UK-wide survey of rehabilitation services in order to gain consensus and a widely accepted definition. They subsequently proposed that rehabilitation is "a whole system approach to recovery from mental ill health which maximizes an individual's quality of life and social inclusion by encouraging their skills, promoting independence and autonomy in order to give them hope for the future and which leads to successful community living through appropriate support',

Rehabilitation potential is broadly defined as the potential to which interventions can optimise and restore function in individuals who have experienced ill-health or the onset of disability [1]. This current paper had initially intended to present a narrative review of the literature around the concept of rehabilitation potential in mental health. However, at present a specific definition of rehabilitation potential relevant to the field of mental health does not appear to exist, which means that there is insufficient literature available on this subject and that the term 'rehabilitation potential' is used with limited consideration as to what is understood by the term. For example, some mental health-focused research articles [9, 16] have acknowledged the concept and use of the term within the field without offering an understanding of this. With this in mind, we must consider as to whether it is feasible for clinicians to make accurate decisions regarding the treatment trajectories of individual's based upon their individual and uniquely-perceived comprehension of the concept of rehabilitation potential in the field of mental health.

\section{Discussion}

In other areas of clinical practice conscious effort has been made to greater understand and define the concept of rehabilitation potential in order to ensure that treatment is appropriately and efficiently allocated to individuals in need of access to rehabilitation services. For example, Burton et al. [2] developed a theoretical model in the field of stroke rehabilitation based upon research findings. They concluded that rehabilitation potential in stroke was defined by observations of individuals achieving their goals, "carry-over" (both within and across treatment sessions) and functional gain. Significantly, the authors describe how rehabilitation potential emerges through the provision (and in some cases, potential failure) of therapy. This not only highlights the importance that rehabilitation provision must be made available to individuals within services for rehabilitation potential to emerge, but that the emerged rehabilitation potential also informs the nature of the provision required in the future. Within the field of dementia, Burton et al. [3] found the concept of carryover to be a mediating factor of an individual's rehabilitation potential as well as poor motivation. It could therefore be considered that rehabilitation potential is a fluid and dynamic process that is sensitive to time and treatment as well as an individual's potentially fluctuating volition. Within the field of acquired brain injury (ABI), Shun et al. [11] conducted research which aimed to identify factors that could influence an occupational therapists' perception of a person's rehabilitation potential following the presence of an ABI. They went on to state that the assessment of rehabilitation potential in the area is a complex process and that when judging an individual's perceived level of rehabilitation potential that therapists should not only consider the patient factors and characteristics. Subsequently, they identified what they termed 'patient related factors' (such as cognitive abilities, physical abilities and pre and post-injury functional status) and 'interpretive activities' (organizational factors, occupational therapists' professional expertise, experiential knowledge, knowledge of scientific evidence and ethical concerns) which aim to 
guide the therapist's perception of an individual's rehabilitation potential.

A challenge of making recommendations around potential service users being deemed suitable for services is that decision-makers (often the multidisciplinary team) will generally attempt to predict each individual's rehabilitation potential, as this is deemed essential in order to maximize the best use of resources [2]. At present, as the concept of rehabilitation potential in mental health is undefined this is largely based upon an individual, or group of individual's personal perceptions of numerous factors, which may not always be considered consistent, valid or reliable indicators of rehabilitation potential in relation to the field of mental health. It is important to consider that one's perception of rehabilitation and ones potential for rehabilitation, can often be the difference in a potential service user accessing rehabilitation services (and/or a more timely discharge) or potentially spending longer periods of time in other services that may not have a rehabilitation focus; thus, potentially resulting in unnecessary and lengthy admissions and preventing these individuals from living more independent and meaningful lives in the community. Crome and Crome [4] warn of this potential outcome by stating that denying access to opportunities for rehabilitation and to work towards personal goals may contribute to an increased risk of admission to longer-term care, which can clearly have implications for the service user, the service and the funding authority. With regards to the funding authority, admissions to rehabilitation services are often lengthy and therefore costly [6, 7] which highlights the need to ensure that all admissions are appropriate.

Without a clear definition of the concept, determining an individual's perceived rehabilitation potential is currently a highly complicated and subjective process. It is important to acknowledge the implications for the clinicians making such important judgments. Lefebvre and Levert [8] have highlighted that it is generally difficult for health care professionals to accurately predict one's rehabilitation potential. From the perspective of staff members within a stroke service entrusted to make decision's around a service user's level of rehabilitation potential, Burton et al. [2] found that staff reported feelings of burden when expected to make decisions around the perceived level of rehabilitation potential displayed by their particular service users.

In addition to the difficulties associated with identifying appropriate candidates for admission to services, pre-admission assessments have proven to be a costly process in terms of both finances as well as resources in that they place demands upon professional teams caring for service users within clinical environments [13, 15]. As such, it is of the upmost importance that assessments provide valid and reliable recommendations and make appropriate choices about an individual's care and rehabilitation needs; as these will essentially provide or deny the individual access to services that offer specific rehabilitation and the chance of living an active and meaningful life. Relating to this point, Goodwin and Allan [5] warn that in the field of dementia care that denying individuals access to rehabilitation can result in reduced wellbeing and quality of life.

When considering the powerful potential of rehabilitation, clinicians should consider the concept discussed in the Burton et al. [2] study, namely that if access to treatment can illicit rehabilitation potential, the individual needs to be able to access these treatments in order for rehabilitation potential to maximised. In addition, to view this in a more pragmatic manner, Goodwin and Allan [5] stated that individuals with the least access to rehabilitation services are actually the ones with most to gain. They go on to suggest that those with the most to gain should be the focus of attention where rehabilitation is concerned; namely ensuring access to rehabilitation services. However, at present those deemed to possess less rehabilitation potential are currently steered towards long-term care.

\section{Conclusion}

The concepts discussed within this piece help to highlight the importance and need for greater understanding around the concept of rehabilitation potential within the field of mental health. At present there is no clear definition of the term when applied specifically to the field, nor is there clarity around the components that are considered to contribute to the concept of rehabilitation potential in mental health. In spite of this, these thoughts have begun to emerge in (but are not limited to) the fields of stroke [2], dementia [3] and 
ABI [11]. Understanding the components that contribute to a sense of rehabilitation potential in mental health will allow for a more universal or widely accepted definition and methods of assessment to be developed which will positively support services and those involved with services. Any measures developed would support the emergence of greater consistency across services when attempting to understand an individual's rehabilitation potential, make decisions around appropriate potential admissions or particular treatment types (including length and duration of treatments). This would also support staff making decisions around potential admission to services or treatments (based upon an individual's perceived rehabilitation potential) and remove some of the burden felt by staff as described by Burton et al. [2].

This piece has also discussed and explored potential implications for service users who are denied access to psychiatric rehabilitation services or for rehabilitative treatment based upon current understanding of the concept of rehabilitation potential in the field of mental health. This can include spending longer periods of time in non, or less rehabilitation-focused services. An obvious risk is that individuals could spend an unnecessary and longer period of time within services due to the lack of presence of rehabilitationfocused treatment [4]. This may also have implications for the individual's perceived feelings of health and well-being [5] as well as their sense of independence. Once comprehensively defined in the field, rehabilitation potential has the potential to ensure that service users are supported appropriately and that the potential for their best future is optimized.

\section{Recommendations}

This paper has identified that there is a clear need for the concept of rehabilitation potential to be better understood in the field of mental health to support services and service users in achieving the best possible outcomes. Further and more extensive research is required with the overall aim of supporting clinicians to better understand the concept of rehabilitation potential. Research should be completed which sets out to comprehensively define the concept of rehabilitation potential in mental health. This would include scoping key parameters of the concept and determining which of these could more accurately predict an individual's rehabilitation potential. This would then support the development of generic measures of rehabilitation potential that could be employed by mental health services to more accurately predict the rehabilitation potential of unique individuals and help to determine the treatment trajectory of these individuals in a more effective manner.

Acknowledgements The authors would like to express their sincere thanks and gratitude to Mrs. Beryl Stanley for her support in this project and sharing of her expertise.

Authors' Contribution The initial idea for this article was formulated by CNE. The literature search and data analysis were completed by CNE and NT. The article was drafted by CNE and critically revised by CNE and NT.Funding Not applicable as there were no funding implications.

\section{Compliance with Ethical Standards}

Conflict of interest None.

Availability of Data and Material Not applicable due to the nature of this piece.

Code Availability Not applicable.

Ethical Approval Not required as this is a theoretical perspective piece.

Open Access This article is licensed under a Creative Commons Attribution 4.0 International License, which permits use, sharing, adaptation, distribution and reproduction in any medium or format, as long as you give appropriate credit to the original author(s) and the source, provide a link to the Creative Commons licence, and indicate if changes were made. The images or other third party material in this article are included in the article's Creative Commons licence, unless indicated otherwise in a credit line to the material. If material is not included in the article's Creative Commons licence and your intended use is not permitted by statutory regulation or exceeds the permitted use, you will need to obtain permission directly from the copyright holder. To view a copy of this licence, visit http://creativecommons.org/licenses/by/4.0/.

\section{References}

1. Bradley G. "Have they got rehabilitation potential?": an ethnographic study exploring meaning and evaluations of rehabilitation potential for an older person following an acute admission' (Ph.D). Northumbria University, Northumbria, United Kingdom, 2015. Available at: https:// ethos.bl.uk/OrderDetails.do?uin=uk.bl.ethos.779871.

2. Burton CR, Horne M, Woodward-Nutt K, Bowen A, Tyrrell P. What is rehabilitation potential? Development of a 
theoretical model through the accounts of healthcare professionals working in stroke rehabilitation services. Disabil Rehabil. 2015;37(21):1955-60.

3. Burton E, Cavalheri V, Adams R, Browne CO, BoverySpencer P, Fenton AM, et al. Effectiveness of exercise programs to reduce falls in older people with dementia living in the community: a systematic review and metaanalysis. Clin Interv Aging. 2015;10:421-34.

4. Crome IB, Crome P. Alcohol and age. Age Ageing. 2018;47:164-7.

5. Goodwin VA, Allan LM. 'Mrs Smith has no rehabilitation potential': does rehabilitation have a role in the management of people with dementia? Age Ageing. 2019;48:5-7.

6. Killaspy H, Harden C, Holloway F, King M. What do mental health rehabilitation services do and what are they for? A national survey in England. J Ment Health. 2005;14:157-65.

7. Killaspy H, Marston L, Green N, Harrison I, Lean M, Holloway F, Craig T, Leavey G, Arbuthnott M, Koeser L, McCrone P, Omar RZ, King M. Clinical outcomes and costs for people with complex psychosis; a naturalistic prospective cohort study of mental health rehabilitation service users in England. BMC Psychiatry. 2016;16(95):1-12.

8. Lefebvre H, Levert M. Breaking the news of traumatic brain injury and incapacities. Brain Inj. 2006;20:711-8.

9. McCreadie RG, Stewart M, Robertson L, Dingwall JM. The Scottish survey of old long-stay in-patients. Br J Psychiatry. 1991;158:398-402.
10. NHS Quality Improvement Scotland. Admissions to adult mental health inpatient services. Edinburgh: NHS Quality Improvement Scotland; 2009.

11. Shun PLW, Bottari C, Ogourtsova T, Swaine B. Exploring factors influencing occupational therapists' perception of patients' rehabilitation potential after acquired brain injury. Aust Occup Ther J. 2017;64:149-58.

12. Sinclair A, Dickinson E. Effective practice in rehabilitation. London: Kings Fund; 1998.

13. Topping-Morris B, Rogers P, Doyle M, Mason T. Pre-admission nursing assessment in a Welsh Medium Secure Unit (1991-2000): Part 2-comparison of traditional nursing assessment with the HCR-20 risk assessment tool. Int J Nurs Stud. 2003;40(6):657-62.

14. Wade DT. What is rehabilitation? an empirical investigation leading to an evidence-based description. Clin Rehabil. 2020;34(5):571-83.

15. Watt A, Topping-Morris B, Mason T, Rogers P. Pre-admission nursing assessment in a Welsh Medium Secure unit (1991-2000): Part 1-an analysis of practice and cost. Int J Nurs Stud. 2003;40(6):645-55.

16. Willis CD. Mental health rehabilitation of detained juveniles: using time wisely. J Am Acad Psychiatry Law. 2011;39(3):150-3.

Publisher's Note Springer Nature remains neutral with regard to jurisdictional claims in published maps and institutional affiliations. 